\title{
How does artificial intelligence in radiology improve efficiency and health outcomes?
}

\author{
Kicky G. van Leeuwen ${ }^{1}$ (D) - Maarten de Rooij ${ }^{1} \cdot$ Steven Schalekamp $^{1} \cdot$ Bram van Ginneken ${ }^{1} \cdot$ Matthieu J. C. M. Rutten ${ }^{1,2}$
}

Received: 31 January 2021 /Revised: 8 April 2021 / Accepted: 24 May 2021 / Published online: 12 June 2021

(C) The Author(s) 2021

\begin{abstract}
Since the introduction of artificial intelligence (AI) in radiology, the promise has been that it will improve health care and reduce costs. Has AI been able to fulfill that promise? We describe six clinical objectives that can be supported by AI: a more efficient workflow, shortened reading time, a reduction of dose and contrast agents, earlier detection of disease, improved diagnostic accuracy and more personalized diagnostics. We provide examples of use cases including the available scientific evidence for its impact based on a hierarchical model of efficacy. We conclude that the market is still maturing and little is known about the contribution of AI to clinical practice. More real-world monitoring of AI in clinical practice is expected to aid in determining the value of $\mathrm{AI}$ and making informed decisions on development, procurement and reimbursement.
\end{abstract}

Keywords Artificial intelligence $\cdot$ Pediatrics $\cdot$ Evidence-based practice $\cdot$ Impact analysis $\cdot$ Innovation $\cdot$ Radiology $\cdot$ Value-based health care

\section{Introduction}

Artificial intelligence (AI) has the potential to change many aspects of health care. However, AI is a means, a tool, not the goal in itself. To create a positive impact on health care with this technology, the clinical goal should be clearly defined. The Da Vinci robot is a well-known example of innovative technology that became very popular very fast, but even today the cost-effectiveness and claim of improved patient outcomes are being debated $[1,2]$. With health care expenses continuously rising through an increasingly older population and evolving technology, we should dare to be critical about what medical devices, including AI-based software, are actually improving health care or making it more efficient.

Kicky G. van Leeuwen

kicky.vanleeuwen@radboudumc.nl

1 Department of Medical Imaging, Radboud University Medical Center, P.O. Box 9101, 6500 HB Nijmegen, The Netherlands

2 Department of Radiology, Jeroen Bosch Hospital, 's-Hertogenbosch, The Netherlands
More than $150 \mathrm{AI}$ products for radiology are on the market [3]. These products have been cleared by the Food and Drug Administration (FDA) or are European Conformity (CE) marked to allow clinical use in the United States and Europe, respectively. Although the supply is large, the scientific evidence on the validation and impact of these products remains limited [4, 5]. A study performed in 2020 showed that only 36 of 100 AI products analyzed had peer-reviewed evidence available on their efficacy [5].

The scientific evidence on the efficacy can be classified according to the hierarchical model developed by Fryback and Thornbury [6] back in 1991 to evaluate the contribution of diagnostic imaging to the patient management process. In a previous study this model was adapted (Table $1 ;[5,6]$ ) to be applicable to assess evidence on AI [5]. The lower levels describe the functioning and performance of the product (levels 1,2). Evaluations regarding higher levels (levels 3-5) describe the impact on the diagnosis, therapy and outcome of the patient. Ultimately, level 6 evidence describes the impact of AI on a macro level, demonstrating the effects on costs and health.

Most studies demonstrated the accuracy of the algorithm (level 2), but (prospective) research showing the benefits in clinical practice (level 3 and up) was limited and covered only 18 of the 100 products evaluated [5]. Another recently published study systematically reviewed evidence on the economic impact of $\mathrm{AI}$ in health care (level 6) and found only six eligible articles 
Table 1 Hierarchical model of efficacy to assess the contribution of artificial intelligence (AI) software to the diagnostic imaging process, adapted from $[5$, 6]

\begin{tabular}{|c|c|}
\hline Level & Explanation \\
\hline \multirow[t]{2}{*}{ Level 1t } & Technical efficacy \\
\hline & Study demonstrates the technical feasibility of the software \\
\hline \multirow[t]{2}{*}{ Level 1c } & Potential clinical efficacy \\
\hline & Study demonstrates the feasibility of the software to be clinically applied \\
\hline \multirow[t]{2}{*}{ Level 2} & Diagnostic accuracy efficacy \\
\hline & Study demonstrates the standalone performance of the software \\
\hline \multirow[t]{2}{*}{ Level 3} & Diagnostic thinking efficacy \\
\hline & Study demonstrates the added value to the diagnosis \\
\hline \multirow[t]{2}{*}{ Level 4} & Therapeutic efficacy \\
\hline & Study demonstrates the impact of the software on the patient management decisions \\
\hline \multirow[t]{2}{*}{ Level 5} & Patient outcome efficacy \\
\hline & Study demonstrates the impact of the software on patient outcomes \\
\hline \multirow[t]{2}{*}{ Level 6} & Societal efficacy \\
\hline & Study demonstrates the impact of the software on society by performing an economic analysis \\
\hline
\end{tabular}

[7], demonstrating the limited evidence of the impact of AI from a more global perspective.

Considering the framework of value-based health care and the corresponding value equation, value=outcome/cost, AI can create value when either reducing the costs or improving the health outcome [8]. We define these as the ultimate goals of AI in radiology, which can be supported by a variety of subgoals described as (1) making the workflow more efficient, (2) shortening the reading time, (3) reducing dose and contrast agents, (4) earlier detection of disease, (5) improved diagnostic accuracy and (6) more personalized diagnostics (Fig. 1).

In this paper, we expand upon the AI tasks and use cases supporting these six objectives, summarized in Table 2, and provide the evidence that demonstrates the (potential) impact of these solutions on health care.

\section{Clinical objectives with artificial intelligence}

\section{More efficient workflow}

With ever increasing health care costs worldwide, effective use of the limited resources is an important endeavor. AI could contribute to this in clinical, but also non-clinical, ways. For example, even before a patient enters the radiology department, AI software might aid the scheduling of imaging appointments and predict no-shows for nudging or more efficient scheduling. Chong et al. [9] trained a model to predict which patients had the highest risk of missing their appointment. These patients received a phone call reminder, decreasing the no-show rate from $19.3 \%$ to $15.9 \%$ [9]. The impact of most of these solutions is not necessarily aimed at the detection or diagnosis of the patient; rather, these solutions address boundary conditions like patient management. The applications, therefore, involve lower risk and have fewer rules and regulations to comply to before they can be implemented in clinical practice. In spite of this, the availability and implementation of such software are limited, leaving room for growth of this industry.

The workflow might also be optimized by changing the diagnostic process with AI. A use case that is already widespread is AI software for tuberculosis detection on chest radiographs. AI-supported tuberculosis detection is especially useful in developing countries where staffing, expertise and financial resources are often limited. This can be used as an autonomous pre-screening tool to reduce the use of microbiological tests,

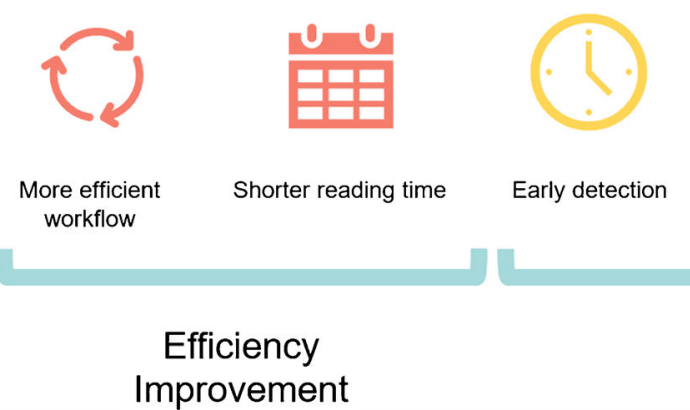

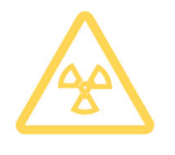

Dose and contrast reduction

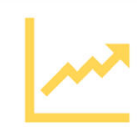

Improved diagnostic accuracy

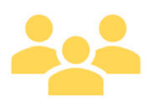

Personalized diagnostics

\section{Increased \\ Health}

Fig. 1 Six objectives that can be pursued with artificial intelligence in radiology to improve efficiency and health outcomes 
which are more time-consuming and costly (levels 2, 3, 6) [10-13]. This is one of the first AI applications in radiology where the software functions autonomously and has taken over the task of the radiologist.

The potential of AI for workflow optimization is also being explored in use cases, but this has not been put into clinical practice. For mammography screening, for example, studies have been performed to simulate an alternative workflow in which an AI risk score determines the number of radiology reads (none, single or double), reducing the total amount of reading time (level 3) $[14,15]$. For lung nodule detection on CT, it has been proposed to empower technicians with AI to leave only some of the workload to the radiologists (level 3) [16].

\section{Shorter reading time}

Apart from increasing diagnostic accuracy and patient outcome, AI can contribute to increasing efficiency of the workforce. Between 2013 and 2018, CT and MR imaging exams increased by $54 \%$ and $48 \%$, respectively, in the United Kingdom, while the radiology workforce grew only $19 \%$ [17]. Increasing numbers of diagnostic imaging examinations along with image technology improvements in the number of slices, reconstructions and sequences have resulted in many more images to review per patient. Decreasing the reading time of the exams could counteract this trend and potentially reduce the rate of burnout for pediatric radiologists as well [18].

Computer-aided detection (CAD) can decrease reading time by making the diagnostic process easier. Research has shown that reading time for normal cases decreased whereas the reading time for pathological cases slightly increased when using CAD tools [19]. Besides quality of the AI system, workflow integration is crucial for making this kind of software a success. Image enhancement could not only shorten image acquisition time but also ease detection, as shown by Martini et al. [20], who found that vessel suppression on CT thorax imaging resulted in a $21 \%$ reduction in reading time for the detection of pulmonary metastasis. Last, the automated quantification of nodules, brain volumes or other tissues, for example, might mitigate some of the tedious manual work that is part of a radiologist's job, along with the large interrater variability inherent to these tasks $[21,22]$. In pediatrics, the automated bone age prediction is a well adopted AI solution aiding the quantification and reading efficiency of hand radiographs [22, 23]. The device could be used autonomously, potentially reducing the reading time to zero, or as a concurrent read to speed quantification.

\section{Early detection}

A timely diagnosis or intervention might be the objective to ultimately improve patient outcome. Especially in critical care situations, such as stroke diagnostics where the phrase "time is 
brain" is used, speed is important and AI has gained ground. AI software is used to analyze CTs and CT angiograms and notify radiologists, hub centers or even the intervention team directly when a large vessel occlusion or intracranial hemorrhage is present. Some preliminary prospective studies have shown the potential positive impact of AI in stroke care by reducing time between the CT angiography and the intervention from $281 \mathrm{~min}$ to $243 \mathrm{~min}$, on average, and reducing length of stay (levels 4, 5) [24, 25].

An alternative method to reduce report turnaround times and promote early detection of critical findings includes worklist prioritization based on urgent findings detected by AI [26]. A study from a German university hospital simulated this concept on retrospective chest radiographs and found that turnaround times for reporting critical findings reduced from $80 \mathrm{~min}$ to $35-50 \mathrm{~min}$ [27]. In the United States, a commercial algorithm to prioritize intracranial hemorrhage resulted in reduced waiting time from $16 \mathrm{~min}$ to $12 \mathrm{~min}$ per positive case [28]. Apart from identifying urgent findings, incidental findings can also be detected early through AI notification. Examples are lung nodule detection on chest radiographs and automated detection of vertebral fractures to identify early signs of osteoporosis using AI applied to every chest or abdomen CT (level 2) [29].

\section{Dose and contrast reduction}

A less renowned goal, but one that is very applicable for AI, is the reduction of radiation dose and intravenous contrast agents. This is even more relevant for pediatric patients because minimizing the use of radiation also minimizes the elevated risk of cancer in younger patients [30]. More frequently, deep learning is being used to advance and speed image reconstruction and post-processing $[31,32]$. Such technology facilitates good image quality with a lower dose or even no dose, as shown by a study from Belgium in which commercial AI software was used to synthesize a CT from MR to assess lesions in the sacroiliac joints for the diagnosis of spondylarthritis, maintaining diagnostic accuracy and making CT potentially redundant (level 3) [33].

\section{Improved diagnostic accuracy}

About half of the AI products for radiology on the market aim primarily at improving diagnostic accuracy [3] by increasing the sensitivity and/or specificity of the diagnostic test. These products are designed to decrease missed diagnoses or prevent unnecessary interventions or examinations, thereby improving health outcomes.

Computer-aided detection algorithms, which were around long before the rise of AI, serve this purpose the most. By reading the exam concurrently with the radiologist or as a second read, bounding boxes, markers and probability scores aid the radiologist in the diagnostic process. Many products are on the market and much research has been conducted to demonstrate the performance of these algorithms in comparison to radiologists or a ground truth (level 2) [13, 34-36]. However, most of these products cannot be used as a standalone medical device, and it is therefore the accuracy of the combination of the software and radiologist that matters (level 3). Considering bone age assessment, researchers demonstrated a substantial increase in diagnostic accuracy for two radiologists aided by $\mathrm{AI}$ software as opposed to using the Greulich-Pyle atlas only (level 3) [22].

Many CAD products are commercially available and have gained ground in clinical practice. These products focus on the detection of cancerous tissue such as breast lesions and lung nodules. However, computer-aided detection is not the only way to reach this objective. Image enhancement and quantitative analysis also support this goal. For example, it has been shown that bone or vessel suppression on thorax imaging can elevate the detection of lung nodules (level 3) [20, 37]. In the area of musculoskeletal radiology, we found that automated knee assessment might improve both the agreement between physicians and the accuracy of the osteoarthritis diagnosis (level 3) [38].

\section{Personalized diagnostics}

Instead of acting based on knowledge and research of a population, AI algorithms can support precision medicine by predicting risks and outcomes based on individual characteristics. This can result in improved health outcomes and aid the allocation of resources, e.g., by providing treatment or additional testing to patients who are expected to benefit most. For example, in the field of neurology, AI-generated brain volume measurements were used to predict the need for further invasive testing to diagnose Alzheimer disease; by using this model, a high diagnostic accuracy could be achieved while only performing additional biomarker testing on $26 \%$ of the population (level 4) [39]. Similarly, for thyroid lesion assessment with US, researchers showed prospectively that biopsies could be avoided when the malignancy risk was assessed with the help of a commercially available AI tool (level 4) [40].

Within the context of breast cancer screening, the classification of breast density aims to personalize the screening process. It is known that women with dense breasts have a higher risk of cancer [41-43]. An automated method makes a stratified screening process feasible in which women with dense breasts receive screening more frequently or with other modalities such as MR imaging. A study from the Netherlands involving more than 40,000 women with extremely dense breast tissue according to commercially available AI software resulted in significantly fewer interval cancers in the group that received additional MR screening (level 5) [42]. 
The use of additional data such as clinical characteristics and genome information could enforce the ability to personalize predictions. However, this is not widespread within the commercially available AI-based radiology software.

\section{Discussion}

Even though the potential of AI to create value to (pediatric) radiology and the patient management process is large, the impact has only been proved in a limited number of cases. Most evidence is based on simulations or retrospective studies. One of the reasons for the lack of evidence might be that the field is still maturing. Most products came to the market only in the last 2 years (Fig. 2) [3]. On average, it takes 17 years for health care innovations to become adopted in clinical practice [44]. Thus, one could argue that AI adoption in clinical practice is still in its infancy.

In the light of pediatric radiology, some applications such as bone age prediction have been widely adopted, but other use cases linger. Vendors tend to go for areas of application that are widely used, are relevant to a large population, and for which there is a large availability of training data ("low hanging fruit"). This causes significant overlap in the clinical use cases addressed by vendors, while many use cases remain unaddressed, including pediatric use cases.

Although FDA clearance and CE marking are present for many AI products, this does not guarantee the added clinical value. The notified bodies (Europe) and FDA (United States) assess the medical devices, including AI software, for risk of harm. Assessing the value and efficacy is up to the vendor and clients themselves, even more so considering that the efficacy is partly hospital-dependent because each hospital's patient populations, imaging brands and workflows are different [45]. Therefore, it has been proposed by multiple parties, including the FDA, to change the way AI software is regulated

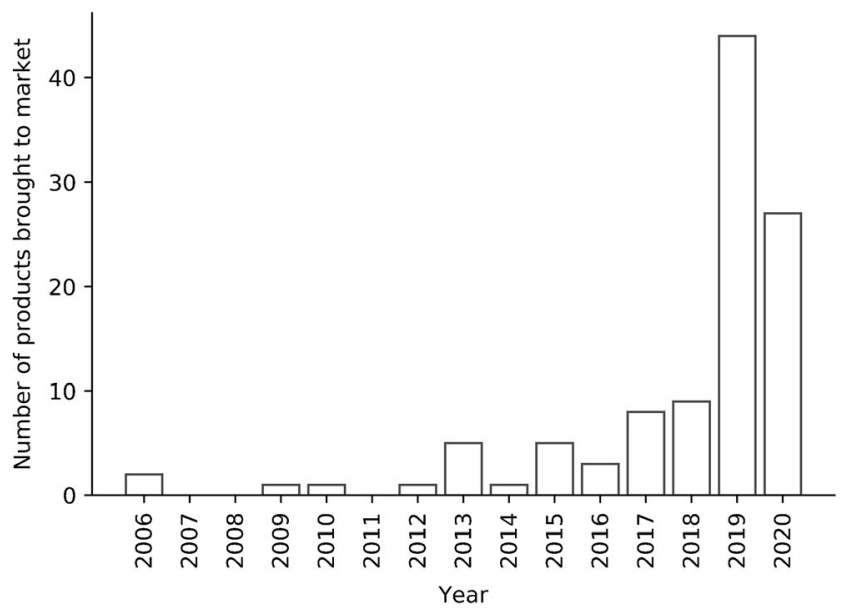

Fig. 2 Number of artificial intelligence products in radiology brought to market based on data from [3] from a standalone evaluation to a more systemic approach where the context of the clinical and human interaction is taken into account [45-47]. This would require more realworld monitoring contributing to the available evidence on the actual clinical impact of AI on health care.

Another challenge for creating clinical value with $\mathrm{AI}$ is the actual technical implementation and deployment of these algorithms. With many narrow task-oriented solutions on the market, the number of contracts and integrations can quickly proliferate and create a large overhead that does not contribute to optimizing efficiency. Further, the increase of information (biomarkers, quantifications, heatmaps, etc.) produced by the algorithms has to be presented in a way that does not decrease efficiency. Marketplaces and mediating platforms have emerged to take some of that burden away. These platforms are still in development and the added value both clinically and financially remains to be studied.

Considering the increased attention on value-based health care, determining the value of a new technology such as AI becomes more relevant for hospitals and insurance companies. With the abundance of AI tools, monitoring or even calculating the expected effects to make informed decisions is crucial. This might demonstrate the return of investment quality or efficiency improvement - justifying the purchase. Monitoring efficacy and use might also minimize the presence of ghost software, or the situation in which software is being paid for but remains unused.

The question remains who is going to pay for the AI tools introduced. Insurance companies might have a role in reimbursing the costs of AI use. In 2002, approval for reimbursement was given in the United States for the use of CAD for mammography, which caused a swift increase in sales and use of the CAD systems [48]. However, this decision backfired as studies by Fenton et al. [49] in 2007 and by Lehman et al. [48] in 2015 showed that the use of these CAD systems in clinical practice did not improve detection rates. Now, the CAD for mammography can no longer be reimbursed separately, making it only profitable to use if the system really improves the efficiency or quality of the tumor detection.

In 2020, Medicare and Medicaid Services took another approach to support the financing and adoption of AI tools in acute stroke using the New Technology Add-on Payment program [50]. The AI solutions aid in the detection of large-vessel occlusions in acute stroke and alert the stroke team to decrease the time to treatment. Preliminary studies have shown that patients might experience less disability and are thus potentially in less need of extra care later in life [24]. These potential benefits mostly lie in the long term, although the costs for the software are made in the short term by, for example, the radiology department. Insurance coverage could be a game changer in AI adoption but requires profound evidence of the clinical impact of the AI tool and use case. Time will tell 
whether this new approval has (re-)opened the gates for other clinical use cases in- and outside the United States.

\section{Conclusion}

Even though the potential of AI software to impact radiology is large, little is known about how it is changing the quality, efficiency and costs of health care. History has shown that real-world validation of these innovations is essential to making informed decisions on further development, procurement, implementation and reimbursement.

Closely evaluating and monitoring the experiences and impact of the AI products in clinical practice should provide insights in its contribution to the initial health care improvement goals. Only then can we prove whether AI is contributing to improved health care with respect to both costs and health outcomes.

\section{Declarations}

\section{Conflicts of interest None}

Open Access This article is licensed under a Creative Commons Attribution 4.0 International License, which permits use, sharing, adaptation, distribution and reproduction in any medium or format, as long as you give appropriate credit to the original author(s) and the source, provide a link to the Creative Commons licence, and indicate if changes were made. The images or other third party material in this article are included in the article's Creative Commons licence, unless indicated otherwise in a credit line to the material. If material is not included in the article's Creative Commons licence and your intended use is not permitted by statutory regulation or exceeds the permitted use, you will need to obtain permission directly from the copyright holder. To view a copy of this licence, visit http://creativecommons.org/licenses/by/4.0/.

\section{References}

1. Crew B (2020) A closer look at a revered robot. Nature 580:S5-S7

2. Wilensky GR (2016) Robotic surgery: an example of when newer is not always better but clearly more expensive. Milbank Q 94:4346

3. Diagnostic Imaging Analysis Group (2020) AI for radiology. Products. Radboud University Medical Center. https://www. aiforradiology.com. Accessed 15 Jan 2021

4. Tariq A, Purkayastha S, Padmanaban GP et al (2020) Current clinical applications of artificial intelligence in radiology and their best supporting evidence. J Am Coll Radiol 17:1371-1381

5. van Leeuwen KG, Schalekamp S, Rutten MJCM et al (2021) Artificial intelligence in radiology: 100 commercially available products and their scientific evidence. Eur Radiol 31:3797-3804. https://doi.org/10.1007/s00330-021-07892-z

6. Fryback DG, Thornbury JR (1991) The efficacy of diagnostic imaging. Med Decis Mak 11:88-94

7. Wolff J, Pauling J, Keck A, Baumbach J (2020) The economic impact of artificial intelligence in health care: systematic review. J Med Internet Res 22:e16866
8. Porter ME (2010) What is value in health care? N Engl J Med 363: 2477-2481

9. Chong LR, Tsai KT, Lee LL et al (2020) Artificial intelligence predictive analytics in the management of outpatient MRI appointment no-shows. AJR Am J Roentgenol 215:1155-1162

10. Khan FA, Majidulla A, Tavaziva G et al (2020) Chest X-ray analysis with deep learning-based software as a triage test for pulmonary tuberculosis: a prospective study of diagnostic accuracy for culture-confirmed disease. Lancet Digit Health 2:e573-e581

11. Murphy K, Habib SS, Zaidi SMA et al (2020) Computer aided detection of tuberculosis on chest radiographs: an evaluation of the CAD4TB v6 system. Sci Rep 10:5492

12. Philipsen RHHM, Sánchez CI, Maduskar P et al (2015) Automated chest-radiography as a triage for Xpert testing in resourceconstrained settings: a prospective study of diagnostic accuracy and costs. Sci Rep 5:12215

13. Qin ZZ, Sander MS, Rai B et al (2019) Using artificial intelligence to read chest radiographs for tuberculosis detection: a multi-site evaluation of the diagnostic accuracy of three deep learning systems. Sci Rep 9:15000

14. Dembrower K, Wåhlin E, Liu Y et al (2020) Effect of artificial intelligence-based triaging of breast cancer screening mammograms on cancer detection and radiologist workload: a retrospective simulation study. Lancet Digit Health 2:e468-e474

15. Lång K, Dustler M, Dahlblom V et al (2021) Identifying normal mammograms in a large screening population using artificial intelligence. Eur Radiol 31:1687-1692. https://doi.org/10.1007/s00330020-07165-1

16. Ritchie AJ, Sanghera C, Jacobs C et al (2016) Computer vision tool and technician as first reader of lung cancer screening CT scans. J Thorac Oncol 11:709-717

17. The Royal College of Radiologists (2018) Clinical radiology UK workforce census report 2018. RCR website. https://www.rcr.ac. uk/publication/clinical-radiology-uk-workforce-census-report2018. Accessed 4 May 2021

18. Desai S (2019) Can artificial intelligence help pediatric radiologist burnout? Imaging Technology News. https://www.itnonline.com/ article/can-artificial-intelligence-help-pediatric-radiologist-burnout. Accessed 3 Dec 2020

19. Rodríguez-Ruiz A, Krupinski E, Mordang J-J et al (2018) Detection of breast cancer with mammography: effect of an artificial intelligence support system. Radiology 290:305-314

20. Martini K, Blüthgen C, Eberhard M et al (2020) Impact of vessel suppressed-CT on diagnostic accuracy in detection of pulmonary metastasis and reading time. Acad Radiol. https://doi.org/10.1016/j. acra.2020.01.014

21. Kim H, Park CM, Hwang EJ et al (2018) Pulmonary subsolid nodules: value of semi-automatic measurement in diagnostic accuracy, diagnostic reproducibility and nodule classification agreement. Eur Radiol 28:2124-2133

22. Kim JR, Shim WH, Yoon HM et al (2017) Computerized bone age estimation using deep learning based program: evaluation of the accuracy and efficiency. AJR Am J of Roentgenol 209:1374-1380

23. Martin DD, Deusch D, Schweizer R et al (2009) Clinical application of automated Greulich-Pyle bone age determination in children with short stature. Pediatr Radiol 39:598-607

24. Hassan AE, Ringheanu VM, Rabah RR et al (2020) Early experience utilizing artificial intelligence shows significant reduction in transfer times and length of stay in a hub and spoke model. Interv Neuroradiol 26:615-622

25. Grunwald IQ, Ragoschke-Schumm A, Kettner M et al (2016) First automated stroke imaging evaluation via electronic Alberta stroke program early CT score in a mobile stroke unit. Cerebrovasc Dis 42:332-338

26. O'Connor SD, Bhalla M (2021) Should artificial intelligence tell radiologists which study to read next? Radiol Artif Intell 3:e210009 
27. Baltruschat I, Steinmeister L, Nickisch H et al (2021) Smart chest $\mathrm{X}$-ray worklist prioritization using artificial intelligence: a clinical workflow simulation. Eur Radiol 31:3837-3845. https://doi.org/10. 1007/s00330-020-07480-7

28. O'Neill TJ, Xi Y, Stehel E et al (2021) Active reprioritization of the reading worklist using artificial intelligence has a beneficial effect on the turnaround time for interpretation of head CT with intracranial hemorrhage. Radiol Artif Intell 3:e200024

29. Dagan N, Elnekave E, Barda N et al (2020) Automated opportunistic osteoporotic fracture risk assessment using computed tomography scans to aid in FRAX underutilization. Nat Med 26:77-82

30. Brody AS, Frush DP, Huda W, Brent RL (2007) Radiation risk to children from computed tomography. Pediatrics 120:677-682

31. Hsieh J, Liu E, Nett B et al (2019) A new era of image reconstruction: TrueFidelity ${ }^{\mathrm{TM}}$ technical white paper on deep learning image reconstruction. GE Healthcare online document. https://www.gehealthcare.com/-/jssmedia/ 040dd213fa89463287155151fdb01922.pdf. Accessed 18 Jan 2021

32. Willemink MJ, Noël PB (2019) The evolution of image reconstruction for CT - from filtered back projection to artificial intelligence. Eur Radiol 29:2185-2195

33. Jans LBO, Chen M, Elewaut D et al (2020) MRI-based synthetic $\mathrm{CT}$ in the detection of structural lesions in patients with suspected sacroiliitis: comparison with MRI. Radiology 298:343-349

34. Alshamrani K, Offiah AC (2020) Applicability of two commonly used bone age assessment methods to twenty-first century UK children. Eur Radiol 30:504-513

35. Chilamkurthy S, Ghosh R, Tanamala S et al (2018) Deep learning algorithms for detection of critical findings in head CT scans: a retrospective study. Lancet 392:2388-2396

36. Rodriguez-Ruiz A, Lång K, Gubern-Merida A et al (2019) Standalone artificial intelligence for breast cancer detection in mammography: comparison with 101 radiologists. J Natl Cancer Inst 111: 916-922

37. Schalekamp S, Karssemeijer N, Cats AM et al (2016) The effect of supplementary bone-suppressed chest radiographs on the assessment of a variety of common pulmonary abnormalities: results of an observer study. J Thorac Imaging 31:119-125

38. Nehrer S, Ljuhar R, Steindl P et al (2019) Automated knee osteoarthritis assessment increases physicians' agreement rate and accuracy: data from the osteoarthritis initiative. Cartilage. https://doi. org/10.1177/1947603519888793

39. Rhodius-Meester HFM, van Maurik IS, Koikkalainen J et al (2020) Selection of memory clinic patients for CSF biomarker assessment can be restricted to a quarter of cases by using computerized decision support, without compromising diagnostic accuracy. PLoS One 15:e0226784

40. Lu Y, Shi XQ, Zhao X et al (2019) Value of computer software for assisting sonographers in the diagnosis of thyroid imaging reporting and data system grade 3 and 4 thyroid space-occupying lesions. J Ultrasound Med 38:3291-3300

41. Astley SM, Harkness EF, Sergeant JC et al (2018) A comparison of five methods of measuring mammographic density: a case-control study. Breast Cancer Res 20:10

42. Bakker MF, de Lange SV, Pijnappel RM et al (2019) Supplemental MRI screening for women with extremely dense breast tissue. N Engl J Med 381:2091-2102

43. French DP, Astley S, Brentnall AR et al (2020) What are the benefits and harms of risk stratified screening as part of the NHS breast screening programme? Study protocol for a multi-site nonrandomised comparison of $\mathrm{BC}$-predict versus usual screening (NCT04359420). BMC Cancer 20:570

44. Hey T, Tansley S, Tolle K (2009) The fourth paradigm: dataintensive scientific discovery. Microsoft website. https://www. microsoft.com/en-us/research/publication/fourth-paradigm-dataintensive-scientific-discovery/. Accessed 4 May 2021

45. Gerke S, Babic B, Evgeniou T, Cohen IG (2020) The need for a system view to regulate artificial intelligence/machine learningbased software as medical device. NPJ Digit Med 3:53

46. Larson DB, Harvey H, Rubin DL et al (2020) Regulatory frameworks for development and evaluation of artificial intelligencebased diagnostic imaging algorithms: summary and recommendations. J Am Coll Radiol 18:413-424

47. United States Food and Drug Administration (2021) Artificial intelligence/machine learning (AI/ML) software as a medical device action plan. FDA website. https:/www.fda.gov/medicaldevices/software-medical-device-samd/artificial-intelligence-andmachine-learning-software-medical-device. Accessed 17 Jan 2021

48. Lehman CD, Wellman RD, Buist DSM et al (2015) Diagnostic accuracy of digital screening mammography with and without computer-aided detection. JAMA Intern Med 175:1828-1837

49. Fenton JJ, Taplin SH, Carney PA et al (2007) Influence of computeraided detection on performance of screening mammography. N Engl J Med 356:1399-1409

50. Hassan AE (2020) New technology add-on payment (NTAP) for Viz LVO: a win for stroke care. J Neurointerv Surg 13:406-408

Publisher's note Springer Nature remains neutral with regard to jurisdictional claims in published maps and institutional affiliations. 